\title{
Hôtel Saint Roch, Albergo Santa Chiara UND Hotel SANTO Domingo: hagIONYMISCH GEBILDETE HOTELNAMEN IN DER ROMANIA
}

\author{
BRIGITTE SEIDLER-LUNZER \\ Wirtschaftsuniversität Wien, Austria \\ HOLGer WOCHele \\ Wirtschaftsuniversität Wien, Austria
}

\begin{abstract}
Hôtel Saint Roch, Albergo Santa Chiara and Hotel Santo Domingo: hagionym-based hotel names in Romance countries
\end{abstract}

\begin{abstract}
Previous articles on hotel names in Romance-speaking countries have shown that hotel names deriving from hagionyms are frequent in France (Giese 1970), Italy (Serianni 1978; Wochele 2007) and Spain as well as in Catalan countries (Herling 2012), whereas they are almost completely inexistent in Romania (Wochele 2009), Germany and Austria. The aim of this research is the analysis of hagionym-based hotel names in the three Romance countries (e.g. regarding high-frequency hagionyms) and to explain their motivation, make an approximate statement about their frequency and contrast these results with the prevalence of hagionyms in other countries (Austria, Germany and Romania).
\end{abstract}

Keywords: hagionyms, hotels, ergonyms, company names, names in business.

\section{Einleitung}

In diesem Beitrag sollen hagionymisch gebildete Hotelnamen in verschiedenen romanischsprachigen Ländern in Europa und im deutschsprachigen Raum vergleichend untersucht werden. Giese 1970 (für Frankreich), Serianni 1978 sowie Wochele 2007 (für Italien) und Herling 2012 (für Mallorca) zeigen, dass dieser Bildungstypus in den drei genannten Ländern nicht unüblich ist. Um in einer vergleichenden Studie diese drei Länder mit dem deutschsprachigen Raum und Rumänien als ostromanischem Land zu kontrastieren, soll zunächst der onymische Status von Hotelnamen geklärt und deren mögliche Kategorisierung (Kapitel 1) dargestellt werden. Nach einer Begriffsklärung des Terminus Hagionym (2) und der Vorstellung der der Studie zu Grunde liegenden Korpora (3) werden die Ergebnisse empirischer Untersuchungen im Hinblick auf Häufigkeit und Verteilung der hagionymenbasierten Hotelnamen für die einzelnen Länder dargestellt (4). Es folgen Hypothesen zur Motivation hagionymenbasierter Hotelnamen und deren gehäuftes Auftreten bzw. Fehlen in einzelnen Ländern. Eine Zusammenfassung, die auch Wege künftiger Forschungen in diesem Bereich aufzeigt, beschließt unseren Beitrag. 
Der onymische Status von Hotelnamen wurde andernorts, z.B. in Wochele (2013: 73f.) schon erörtert, weswegen hier nicht näher darauf eingegangen werden soll. Hotelnamen werden den Ergonymen und nicht den Toponymen zugerechnet, da bei ihnen nicht die orientierende Funktion (Ortsnamen), sondern die werbende Funktion im Vordergrund steht; auch bleibt der Name des Hotels nicht an ein bestimmtes Gebäude gebunden. Im Falle einer Übersiedlung kann er einfach „mitgenommen“ werden. Fahlbusch 2015: 286-287 macht eine weitere wichtige Unterscheidung, die auch für Hotelnamen relevant ist: Hotels tragen häufig einen GeschäftsN oder EtablissementN, der für die einzelnen Niederlassungen gilt, und vom eigentlichen, offiziellen Namen der Unternehmens, das eine bestimmte Hotelkette betreibt, abweichen kann (Hotelnamen: Frankfurter Hof, Frankfurt/Main; Steigenberger Hotel Graf Zeppelin, Stuttgart vs. Unternehmensname Steigenberger Hotels AG).

Weiters besteht die Tendenz, die Zugehörigkeit von Hotels zu bestimmten Hotelketten oder Hotelmarken mittels Markennamen im Hotelnamen zum Ausdruck zu bringen und als Bestimmungswort ein Toponym oder Mikrotoponym hinzuzufügen: Hôtel Ibis Gare de Lyon Diderot (Wochele 2017: 189). Dies führt zu langen, asyndetisch gereihten Nominalphrasen als Hotelnamen, wobei sich aus Marketingsicht empfiehlt, das Hotelangebot eines Unternehmens durch Kreation verschiedener Untermarken zu differenzieren: „Je mehr Untermarken man erfindet, desto weniger anonym wirkt der Bettenkonzern. Weshalb es zum Beispiel Grand Hyatt, Park Hyatt und Hyatt House gibt [...]“" (Krüger 2015).

Im Zusammenhang mit dem konkreten Hotelnamen sei auch nochmals auf die schon andernorts erwähnte (Wochele 2017: 186) Problematik hingewiesen, dass der Name des einzelnen Hotelbetriebs je nach Internet-Suchplattform, gedrucktem Verzeichnis oder Homepage des Hotels variieren kann - vor allem in Hinblick auf die Setzung oder Nicht-Setzung des Bestimmungsworts Hotel als erstem Element der komplexen Nominalphrase „Hotelname“.

\section{Kategorisierung von Hotelnamen}

Die sprachliche Analyse von HotelN kann auf verschiedenen Ebenen geschehen. Zunächst kann man Auffälligkeiten auf der graphisch-phonetischen Ebene untersuchen. Mittels so genannter Attraktoren versuchen Hotelbesitzer die Aufmerksamkeit potenzieller Gäste zu erregen; ein gutes Beispiel hierfür wäre das Innsbrucker Designhotel aDLERS. Allerdings ist festzustellen, dass die genauen Schreibungen von Hotelnamen wie gerade dargelegt häufig stark variieren - und dadurch Aussagen über graphische Attraktoren nur mit gewissen Vorbehalten getroffen werden können. Man kann weiters die morphologische oder die semantische Struktur von HotelN untersuchen: Auch wenn Eigennamen keine lexikalische Bedeutung haben, sondern lediglich auf einen Referenten verweisen, kann man untersuchen, aus welchen lexikalischen oder onymischen Elementen Waren- oder Unternehmensnamen aufgebaut sind - und welche „Spenderbezirke“ besonders dabei häufig sind, sofern es sich nicht um „Kunstwörter“ oder „Konzeptformen“ handelt (Platen 1997: 41-45). 
Serianni 1978 hat auf der Grundlage eines italienischen Korpus eine sehr vollständige Kategorisierung der Namenbasen von HotelN vorgenommen, die für andere Studien geringfügig erweitert und angepasst wurde (Wochele 2007: 320-323) und 22 verschiedene Kategorien umfasst. Nur kurz erwähnt sei hier, dass viele HotelN nicht eindeutig nur einer bestimmten Kategorie zuzuordnen sind: So kann das Ferdinand Hotel in Rumänien (Constanța) der Kategorie E2 (,Männlicher Vorname') zugewiesen werden, oder aber auch der Kategorie F (,Name einer berühmten Persönlichkeit'), da es eine Reverenz an König Ferdinand I. von Rumänien (1865-1927) sein könnte. Um die zutreffende Etymologie herauszufinden, wären für jeden Einzelfall genauere Recherchen notwendig. Dies ist aber gar nicht Ziel dieser Untersuchung - ebenso wenig wie der Umstand eine Rolle spielt, dass ein bestimmter Prozentsatz von HotelN überhaupt keiner Kategorie von Serianni 1978 oder Wochele 2007 zugewiesen werden können (z.B. Hôtel Le M, Paris). Dies gilt beispielsweise für die erwähnten Konzeptformen oder Phantasienamen. In diesem Zusammenhang ist auch erwähnenswert, dass Herling 2012 für ihr Korpus einen Prozentsatz von 12,35 \% nicht eindeutig kategorisierbaren Materials angibt (2012:210). Gegenstand dieses Beitrags soll jedoch die Kategorie $\mathrm{H}$, und zwar hagionymisch basierte HotelN sein, wie die schon im Titel genannten Hotelnamen Hôtel Saint Roch (Paris, Avignon usw.), Albergo Santa Chiara (Rom) und Hotel Santo Domingo (Madrid).

\section{Hagionyme und hagionymenbasierte Hotelnamen}

Aus forschungspraktischen sowie wissenschaftstheoretischen Gründen scheint es plausibel, wenn nicht gar banal, bei einer Untersuchung zu hagionymenbasierten HotelN nach einer Definition des Terminus Hagionym zu suchen. Obschon bei den meisten SprecherInnen ein intuitives Verständnis davon vorhanden sein dürfte, was ein Heiligenname ist - erweist es sich tatsächlich als schwierig, eine Definition von Hagionym oder Heiligennamen ausfindig zu machen. Debus 2012 erwähnt beispielsweise lediglich, wie Namen von Heiligen die Rufnamengebung in Deutschland beeinflussten (2012: 87), ähnliche Ausführungen finden sich bei Nübling et al. (2015: 115) oder Koß (2002: 129f. und 135). Man kann also Greule 2016 zustimmen: „Nach einer Definition von HeiligenN sucht man vergeblich" (Greule 2016: 295).

Was macht einen gewöhnlichen, „normalen“ Namen zu einen Heiligennamen? Laut Greule 2016: 295 erhalten PersonenN das Attribut sanctus/heilig, wenn sie im Heiligenverzeichnis Martyrologium Romanum oder im Ökumenischen Heiligenkalender verzeichnet sind. In diesem Heiligenverzeichnis sind 6650 Heilige und Selige und 7 500 Märtyrer gelistet.

Folgt man Greule 2016 weiter, so gilt als „Signal der Transposition eines Lexems zum HeiligenN [...] das präponierte Adjektiv heilig, im Plural heilige" (Greule 2016: 293). Das heißt forschungspraktisch für die vorliegende Studie, dass aus den im folgenden Kapitel vorgestellten Korpora diejenigen Hotelnamen extrahiert wurden, in denen das attributive Adjektiv Sankt, it./span. Santo/Santa bzw. San, frz. Saint/Sainte und rum. Sfint/Sfinta vor einem männlichen oder weiblichen Rufnamen steht. Im 
Umkehrschluss bedeutet dies, dass auch in denjenigen Fällen, in denen der HotelN zwar einen Rufnamen enthält, der im Martyrologium Romanum verzeichnet ist, ihm jedoch nicht das attributive Adjektiv Sankt, Sfânt etc. vorangestellt ist, dieser Hotelname nicht in unsere Korpora aufgenommen wurde. So wurde das Stephanushaus, das von den Benediktinerinnen des Unbefleckten Herzens Mariens betrieben wird, nicht in das österreichische Korpus aufgenommen - da eben das Adjektiv Sankt fehlt, und zudem das Haus nicht als Hotel betrieben wird.

\section{HotelN-Korpora}

Für die Erstellung der Korpora wurden ausschließlich die Kategorie Hotel berücksichtigt, d.h. keine Pensionen, keine Privatquartiere, Ferienhäuser, Campingplätze, Kurheime, Jugendherbergen, Schutzhütten, Kinder- und Jugenderholungsheime oder Matratzenlager. Zu den Charakteristiken eines Hotels zählen vor allem das Vorhandensein einer Rezeption und einer größeren Anzahl von Zimmern, einer Bar oder eines Restaurants und andere Annehmlichkeiten, deren Standard nach Sternen bewertet wird (cf. Kriterienkatalog Hotelklassifizierung ${ }^{1}$ ).

\subsection{Frankreich}

In Frankreich kann laut INSEE $2017^{2}$ im Jahr 2016 von einer Gesamtzahl von ca. 18.300 Hotels ausgegangen werden. Um ein Korpus von Hotelnamen zu erstellen, die ein Hagionym enthalten, wurde die Interplattform Holiday.check ${ }^{3}$ verwendet und die Internetausgabe der pages jaunes ${ }^{4}$, dem offizielle Firmen- und Branchenverzeichnis Frankreichs im Telefonbuch, wobei bei diesem zweiten Schritt der Verifizierung stichprobenartig auf eine breite geographische Streuung der Belege nach Region bzw. StadtLand geachtet wurde. Auf der Grundlage dieser Ergebnisse wurde eine Datenbank mit 463 Einträgen angelegt.

\subsection{Italien}

Die Grundlage des italienischen Korpus war das Annuario Alberghi d'Italia 2005, ein gedrucktes offizielles Verzeichnis der italienischen Hotels; es kann davon ausgegangen werden, dass sich seitdem die Anzahl der hagionymenbasierten HotelN, wenn überhaupt, nur sehr geringfügig geändert haben dürfte. Laut Annuario belief sich in Italien die Anzahl der Hotels im Jahr 2005 auf 31.997.

\subsection{Rumänien}

Für Rumänien wurde gleichfalls ein etwas älterer, gedruckter Führer, der Hotel Guide 2006, verwendet, der offiziellen Charakter hat und vom ,Ministry of Transports,

1 https://www.wko.at/site/hotelsterne/Kriterienkatalog-Hotelklassifizierung-20152020.pdf (letzter Zugriff 29.09.2017).

2 www.insee.fr/fr/statistiques/1283850\#documentation (letzter Zugriff am 23.08.2017).

www.holidaycheck.de (letzter Zugriff 28.08.2017).

4 https://mesannuaires.pagesjaunes.fr/ (letzter Zugriff: 27.08.2017). 
Constructions and Tourism, National Authority of Tourism' herausgegeben wurde. Er beansprucht Vollständigkeit: „This guide includes a comprehensive list with the hotel premises from all over the country" (Hotel Guide 2006: 3). Wenn man die dort gelisteten Hotels auszählt, kommt man auf eine Gesamtzahl von 904 Hotels. Allerdings dürfen gemäß Bugheşiu 2013 Zweifel an der Vollständigkeit gehegt werden. Die Konsultation von Booking.com hat eine Gesamtzahl von 6.252 Unterkünften für Rumänien ergeben, wobei hier allerdings auch Pensionen und B\&B gelistet sind.

\subsection{Spanien}

Für das spanische Korpus wurde eine Stichprobe für Madrid über Booking. $\mathrm{com}^{5}$ durchgeführt. Das Ergebnis waren 1.660 Hotels in Madrid (28.08.2017), auf den páginas amarillas im Telefonbuch werden für Madrid-Zentrum 397 Hotels gelistet $^{6}$. Ergänzend wird die Untersuchung von Herling 2012 herangezogen, wo Namen von 534 Unterkünften auf Mallorca analysiert werden.

\subsection{Deutschland}

Das Portal für Hotellerie, Gastronomie und deren Branchen, Hotelier.de ${ }^{7}$, verzeichnet im Jahr 2015 in dem synchron vorhandenen Korpus insgesamt an die 15.000 Hotels in Deutschland. Die Suche wurde über die Internetplattform Holidaycheck.at und stichprobenartig über Booking.com ergänzt.

\section{6. Österreich}

In Österreich werden von Statistik Austria ${ }^{8}$ für die Jahre 2015/16 insgesamt 12.400 Hotels angeführt. Zur Korpuserstellung wurde auf Holidaycheck.at und punktuell auch über Booking.com nach hagionymenbasierten HotelN gesucht; des Weiteren erwies sich das österreichweite Hotelverzeichnis auf Schnelle-Hotelsuche.at ${ }^{9}$ als sehr nützlich, da es eine alphabetische Auflistung nach Bundesländern ausschließlich von Hotels ab 3 Sternen ermöglicht.

\section{Ergebnisse und ihre Distribution}

\subsection{Frankreich}

Die zahlenmäßige Auswertung der hagionymenbasierten HotelN am Gesamtkorpus ergibt 463 Einträge von HotelN mit „Saint/e“, was einem Anteil von ca. 2,5\% in Bezug auf insgesamt 18.300 Hotels in Frankreich entspricht. Die Anzahl der

\footnotetext{
5 www.booking.com (letzter Zugriff 02.09.2017).

6 https://www.paginasamarillas.es/search/alojamientos/all-ma/madrid/all-is/madrid/ all-ba/all-pu/all-nc/hoteles/all-ct/27?what=hoteles\&where=madrid (letzter Zugriff 28.08.2017).

7 www.hotelier.de (letzter 18.08.2017).

8 https://de.statista.com/statistik/daten/studie/294862/umfrage/anzahl-der-beherbergungsbetriebe-in-oesterreich/ (letzter Zugriff 18.08.2017).

9 http://www.schnelle-hotelsuche.at (letzter Zugriff 24.08.2017).
} 
unterschiedlichen Hagionyme in diesen 463 Belegen (Token) beläuft sich insgesamt auf 141 HeiligenN (Types), mit 132 männlichen (93,6 \%) und 9 weiblichen (6,3\%) Hagionymen.

Tab. 1 Männliche HeiligenN in Frankreich

\begin{tabular}{|l|c|c|}
\hline $\begin{array}{l}\text { TOP 10 der männlichen HeiligenN } \\
\text { (gesamt 132) }\end{array}$ & \multicolumn{2}{|c|}{$\begin{array}{c}\text { Anzahl der entsprechenden HotelN } \\
(463=100 \%)\end{array}$} \\
\hline 1. Saint Germain (inkl. toponym. Ergänzungen) & 35 & $7,5 \%$ \\
\hline 2. Saint Martin (inkl. toponym. Ergänzungen) & 21 & $4,5 \%$ \\
\hline 3. Saint Jean (inkl. toponym. Ergänzungen) & 20 & $4,3 \%$ \\
\hline 4. Saint Charles & 19 & $4,1 \%$ \\
\hline 5. Saint Louis (inkl. toponym. Ergänzungen) & 19 & $4,1 \%$ \\
\hline 6. Saint Michel (inkl. toponym. Ergänzungen) & 18 & $3,6 \%$ \\
\hline 7. Saint Georges & 17 & $3,6 \%$ \\
\hline 8. Saint Antoine & 13 & $2,8 \%$ \\
\hline 9. Saint Paul & 12 & $2,3 \%$ \\
\hline 10. Saint Malo & 10 & $2,1 \%$ \\
\hline
\end{tabular}

Das regionale Vorkommen der einzelnen HeiligenN richtet sich - abgesehen von Universalheiligen - generell nach den Orten, in denen sie gelebt und gewirkt haben ${ }^{10}$. An erster Stelle der großen französischen Heiligen, die in Hotelnamen vertreten sind, findet man Saint Germain, der von 550-576 Bischof von Paris war. Die entsprechenden Hotelbenennungen sind natürlich toponymisch begründet - sei es aufgrund ihrer Lage im Pariser Stadtviertel Saint-Germain-des-Prés oder in einer der fünf Gemeinden Saint-Germain oder der 245 Orte Saint-Germain mit Namenszusatz ${ }^{11}$. An zweiter Stelle kommt Saint Martin, Bischof in Tours, wo er allerdings in keinem HotelN vertreten ist ${ }^{12}$. Saint Charles ist in und um Marseille sehr präsent, Saint Jean in und um Bordeaux, Saint Louis - entweder der französische König Ludwig IX., der Heilige, oder der Erzbischof von Toulouse - war Namengeber von Hotels in der Region um Paris und im Süden Frankreichs. Saint Michel ist häufig in HotelN in der Normandie anzutreffen und Saint Georges in Burgund, in der Region Nuits-Saint-Georges. HotelN mit Saint Antoine, Saint Paul sind toponymisch bedingt ausschließlich im Süden Frankreichs zu finden, Saint Malo nur in der Bretagne.

Nicht berücksichtigt wurden acht HotelN, die das Hagionym Saint Exupéry enthalten und die sich im Süden Frankreichs und um Lyon, dem Geburtsort von Antoine de Saint Exupéry befinden. Es liegt nahe, dass diese Hotels nach dem berühmten

10 Leroquais (zit. in Arx 1970: 128) teilt die Heiligen in drei Kategorien ein: „Universalheilige, die in der ganzen Kirche Verehrung genießen; Regionalheilige, die in einem Spezialkult einer bestimmten Gegend verehrt werden und Lokalheilige, deren Verehrung auf eine kleiner Gruppe von Landschaften oder Orten beschränkt ist".

11 https://de.wikipedia.org/wiki/St._Germain (letzter Zugriff 28.09.2017).

12 In Tours findet man jedoch bloß die HotelN Saint-Jean und Saint-Eloi. 
Schriftsteller benannt wurden und nicht nach dem Heiligen Exuperius von Toulouse, wobei allerdings anzumerken ist, dass der Familienname auf den Ort Saint-Exupéry im Limousin zurückgeht, der wiederum nach dem Heiligen Exuperius benannt ist (cf. Marck 2012).

Die weiblichen Hagionyme mit elf Token und neun Types werden von Sainte Marie (drei Mal) angeführt und sind vorwiegend in Lourdes und im Süd-Osten Frankreichs vertreten.

Tab. 2 Weibliche HeiligenN in Frankreich

\begin{tabular}{|l|l|l|}
\hline $\begin{array}{l}\text { Weiblich HeiligeN } \\
\text { gesamt }\end{array}$ & Ort & Region \\
\hline Sainte Agnès & Lourdes & Occitanie \\
\hline Sainte Bernadette & Lourdes & Occitanie \\
\hline Sainte Catherine & Lourdes & Occitanie \\
\hline Sainte Honorine & Conflans-Sainte-Honorine & Île-de-France \\
\hline Sainte Marie & Lourdes & Occitanie \\
\hline Sainte Marie & Nolay & Bourgogne \\
\hline Sainte Marie-la-Blanche & Sainte-Marie-la-Blanche & Bourgogne \\
\hline Sainte Marthe & Avignon & Provence-Alpes-Côte d'Azur \\
\hline Sainte Odile & Obernai & Hauts-de-France \\
\hline Sainte Rose & Lourdes & Occitanie \\
\hline Sainte Sabine & Saint-Jean-de-Losne & Bourgogne \\
\hline
\end{tabular}

Generell kann festgestellt werden, dass in Frankreich die große Mehrheit der Hotels mit hagionymenbasierten Namen gute Mittelklassehotels sind. Unter den 100 im Jahr 2010 mit 5 Sternen gelisteten Hotels ${ }^{13}$ findet man 13 mit Heiligennamen, die mehrheitlich auf ein Toponym zurückgehen, ansonsten sind sie der Analysekategorie I nach Serianni 1978 zuzuordnen: Appellative bzw. Adjektive, die auf eine königliche, aristokratische oder nicht-adelige Elite verweisen, die die Kundschaft des betreffenden Hotels darstellt oder darstellen soll (z.B, Regency, Royal Riviera, Château, Princesse, Palace).

1. Hôtel La Bastide Saint-Antoine (Grasse)

2. Hôtel Le Saint Paul (Saint-Paul-de-Vence)

3. Château Saint-Martin et Spa (Vence)

4. L'Ermitage Mont Saint Michel (Beauvoir)

5. Le Saint Roch (Courchevel)

6. Le Saint Joseph (Courchevel)

7. Esprit Saint-Germain (Paris $6^{\mathrm{e}}$ )

8. Hôtes San Regis (Paris $8^{\mathrm{e}}$ )

9. Villa Marie Saint-Tropez (Ramatuelle)

13 https://fr.wikipedia.org/wiki/Liste_des_h\%C3\%B4tels_class\%C3\%A9s_ cinq_\%C3\%A9toiles_en_France (letzter Zugriff am 25.09.2017). 
10. Hôtel La Bastide de Saint-Tropez (Saint-Tropez)

11. Hôtel La Tartane Saint-Amour (Saint-Tropez)

12. Hôtel Sezz Saint-Tropez (Saint-Tropez)

13. Hôtel La Côte Saint-Jacques (Joigny)

\subsection{Italien}

Es wurde ein geographisch umfassendes Korpus von 136 Einträgen auf der Grundlage des Annuario Alberghi d'Italia 2005 erstellt, das eine Auswahl an Städten und Orten aus allen Regionen Italiens berücksichtigt. Der Anteil dieser 136 Token macht 2,9\% des Gesamtkorpus aus (cf. Wochele 2007: 323); sie enthalten 65 Types, darunter 50 männliche HeiligenN (76,9 \%) und 15 weibliche HeiligenN (23\%). Eine stichprobenartige Auswertung für Rom ergab insgesamt 755 Hotels, von denen 22 hagionymenbasiert sind. Die Top 10 der italienischen HeiligenN werden von San Marco angeführt:

Tab. 3 Männliche HeiligenN in Italien

\begin{tabular}{|l|c|}
\hline $\begin{array}{l}\text { TOP 10 der männlichen HeiligenN } \\
\text { gesamt 50) }\end{array}$ & $\begin{array}{c}\text { Anzahl der entsprechenden HotelN } \\
\text { (gesamt 136) }\end{array}$ \\
\hline 1. San Marco & 13 \\
\hline 2. San Giorgio & 7 \\
\hline 3. San Carlo & 6 \\
\hline 4. San Michele & 6 \\
\hline 5. San Paolo & 6 \\
\hline 6. San Remo & 4 \\
\hline 7. San Pietro & 3 \\
\hline 8. San Francesco & 3 \\
\hline 9. San Lorenzo & 3 \\
\hline 10. San Giovanni & 2 \\
\hline
\end{tabular}

Bei den weiblichen HeiligenN steht in Italien - wie in Frankreich - die Heilige Maria an erster Stelle. Es ist interessant, dass es in Italien mit insgesamt 15 weiblichen Hagionymen fast doppelt so viele wie in Frankreich gibt. Nur 5 unterschiedliche weibliche HeiligenN sind mehr als ein Mal Namensgeberinnen für ein Hotel.

Tab. 4 Weibliche HeiligenN in Italien

\begin{tabular}{|l|c|}
\hline $\begin{array}{l}\text { TOP 5 der weiblichen HeiligenN } \\
\text { (gesamt 15) }\end{array}$ & $\begin{array}{c}\text { Anzahl der entsprechenden HotelN } \\
\text { (gesamt 136) }\end{array}$ \\
\hline 1. Santa Maria & 7 \\
\hline 2. Santa Chiara & 4 \\
\hline 3. Santa Lucia & 4 \\
\hline 4. Santa Caterina & 2 \\
\hline 5. Santa Maura & 2 \\
\hline
\end{tabular}




\subsection{Spanien}

Herling 2012: 207 listet folgende hagionymenbasierte HotelN für Mallorca auf: Hotel San Diego, Hotel Sant Salvador, Hotel Santa Lucia, Hotel San Lorenzo, Casal Santa Eulàlia, Hotel Santa Ana, Hotel Sant Jordi ('Heiliger Georg'), Hotel La Santa María etc. ${ }^{14}$ (d.h. mindestens 8 von 534, quantitative Angaben können infolgedessen nicht gemacht werden).

Stichprobenartige Untersuchungen für Madrid ergaben folgende hagionymenbasierten Namen in der spanischen Hauptstadt: Ac Hotel Santo Mauro Autograph Collection, Hotel Santo Domingo, Hotel JC Rooms Santa Ana, Hotel Intur Palacio San Martin, Petit Palace San Bernardo, Petit Palace Santa Barbara, (Santander) - d.h. sechs Belege für 397 Hotels, was einem Anteil von 1,5\% entspricht.

\subsection{Rumänien}

Generell finden man in Rumänien keine hagionymenbasierten Hotelnamen, es sei denn, man berücksichtig auch das Best Western Hotel Park Sfântu Gheorghe in Sfântu Gheorghe (Covasna); eine Pension (lt. Booking.com) Sfânta Maria in Comandău (Covasna) und Casa de oaspeți (,Gästehaus') Sfântu Nicolae in Iaşi. Wochele 2007: 323 nennt zwei Belege für hagionymisch basierte HotelN in Rumänien, was einem Anteil von $0,2 \%$ am Gesamtkorpus entspricht. Bezieht man die Pension und das Gästehaus mit ein, kommt man - auch wenn man nur 904 Hotels als Gesamtkorpus zu Grunde legt - auf 0,3 \%, bei den Angaben der Plattform Booking.com käme man sogar auf nur $0,047 \%$.

\subsection{Deutschland}

Das synchron vorhandene Korpus an Hotelnamen führt im Jahr 2015 ca. 15.000 Hotels in Deutschland laut der Internetplattform Hotelier.de ${ }^{15}$ an. In Relation zu unseren Untersuchungsergebnissen von 31 Belegen an hagionymenbasierten HotelN kommt man auf einen gleichfalls verschwindend geringen Anteil von $0,2 \%$. Diese 31 Token enthalten 19 Types, davon 18 männliche und 1 einzigen weiblichen HeiligenN (Hotel St. Annen in Hamburg). Das Hotel Santo in Karlsruhe wurde nicht berücksichtigt, da es keinen EigenN enthält.

14 Es sei an dieser Stelle auf die Problematik hingewiesen, dass der Benennung auch ein Toponym oder eine nahe gelegene Kirche mit dem jeweiligen Heiligennamen zugrunde liegen kann.

15 http://www.hotelier.de/hotellerie/hotelgewerbe/38737-hotelmarkt-deutschland-2013-2012-2011-2010-2009-hotelgewerbe-news (letzter Zugriff: 18.08.2017). Die Gesamtzahl der Beherbergungsbetriebe in Deutschland wird demgegenüber von https://de.statista. $\mathrm{com} /$ statistik/daten/studie/29627/umfrage/geoeffnete-beherbergungsbetriebe-in-deutschland-seit-1992/ (letzter Zugriff 18.08.2017) mit 50.728 für das Jahr 2016 angegeben. Diese Zahl beinhaltet allerdings auch Privatunterkünfte, Ferienwohnungen, Kurheime, Pensionen, Campingplätze, Jugendherbergen etc. Im Artikel zu den beliebtesten Hotelnamen (s. nächste Fußnote) werden bedauerlicherweise keine quantitativen Angaben zum Gesamtkorpus gemacht. 
Tab. 5 Männliche HeiligenN in Deutschland

\begin{tabular}{|l|c|}
\hline $\begin{array}{l}\text { TOP 10 der männlichen HeiligenN } \\
\text { (gesamt 18) }\end{array}$ & $\begin{array}{c}\text { Anzahl der entsprechenden HotelN } \\
\text { (gesamt 31) }\end{array}$ \\
\hline 1. Heiliger Georg & 6 \\
\hline 2. Heiliger Josef / Joseph & 4 \\
\hline 3. Heiliger Andreas & 2 \\
\hline 4. Heiliger Laurentius & 2 \\
\hline 5. Heiliger Martin & 2 \\
\hline 6. Heiliger Pauli & 2 \\
\hline 7. Heiliger Andrä & 1 \\
\hline 8. Heiliger Englmar & 1 \\
\hline 9. Heiliger Florian & 1 \\
\hline 10. Heiliger Fridolin & 1 \\
\hline
\end{tabular}

\section{6. Österreich}

In Österreich kann eine Maximalzahl von 16 hagionymenbasierten HotelN angesetzt werden; in Bezug zum Gesamtkorpus von 12.400 Hotels im Jahr 2016 (laut Statistik Austria ${ }^{16}$ ) entspricht das einem Anteil von $0,13 \%$. Auf diese 16 Hotels entfallen 12 ausschließlich männliche HeiligenN, bei einem Namen handelt es sich um einen PhantasieN.

Tab. 6 Männliche HeiligenN in Österreich

\begin{tabular}{|l|c|}
\hline $\begin{array}{l}\text { TOP 10 der männlichen HeiligenN } \\
\text { (insgesamt 12) }\end{array}$ & $\begin{array}{c}\text { Anzahl der entsprechenden HotelN } \\
\text { (insgesamt 16) }\end{array}$ \\
\hline 1. Heiliger Georg / Georgen & 3 \\
\hline 2. Heiliger Hubertus & 2 \\
\hline 3. Heiliger Daniel & 1 \\
\hline 4. Heiliger Lambrecht & 1 \\
\hline 5. Heiliger Laurentius & 1 \\
\hline 6. Heiliger Martin & 1 \\
\hline 7. Heiliger Marx & 1 \\
\hline 8. Heiliger Michael & 1 \\
\hline 9. Heiliger Nikolaus & 1 \\
\hline 10. Heiliger Oswald & 1 \\
\hline
\end{tabular}

Interessant ist für Österreich, dass es in der Hauptstadt Wien nur 2 hagionymenbasierte HotelN gibt. Der Name des einen Hotels ist ibis Budget Hotel Sankt Marx, das den Namen des Stadtteils trägt, der auf eine dem heiligen Markus geweihte Kapelle zurückgeht. Der Name des zweiten Hotels, Saint Shermin, mutet heilig an, ist es aber

16 https://de.statista.com/statistik/daten/studie/294862/umfrage/anzahl-der-beherbergungsbetriebe-in-oesterreich/ (letzter Zugriff 18.08.2017). 
nicht. „Shermin“ ist ein persischer weiblicher Vorname („die Schüchterne“) und geht auf die erste Besitzerin zurück. Bei der Neuübernahme sollte das Hotel auch einen neuen Touch bekommen, der ursprüngliche Namen aufgrund der besonders leichteren Auffindbarkeit (bei der Suche mit Google erscheint Hotel Shermin immer an erster Stelle) aber beibehalten werden. So wurde von der neuen Besitzerin ein "Saint“ vorangestellt - ohne jeglichen Bezug zu einem HeiligenN. Unbeabsichtigt ergab sich - bei französisierter Aussprache von Shermin - eine phonetische Ähnlichkeit mit dem Pariser Stadtviertel Saint-Germain-des-Prés, und diese Assoziation, die durch den Zusatz „\& Champagne“ nach „Bed, Breakfast“ verstärkt wird, wird unbewusst-bewusst gern in Kauf genommen.

Generell ist in Bezug auf Österreich zu erwähnen, dass es in jedem der neun Bundesländer nur zwei bis drei hagionymenbasierte Hotels gibt. Es handelt sich dabei - im Gegensatz zu Frankreich und Italien - eher um luxuriöse Hotels (ab 3 Sternen), deren exklusiver Name mit hoher Qualität gleichgesetzt werden soll.

Zusammenfassend kann also festgestellt werden, dass HotelN, die auf Heiligennamen basieren, einen relativ hohen Anteil in Italien, Frankreich und Spanien haben, während ihr Anteil in Deutschland, Österreich und Rumänien verschwindend klein ist (ca. 10-mal niedriger). Wichtig ist nochmals zu betonen, dass es sich bei allen zahlenmäßigen Angaben in unserer Untersuchung um ungefähre Näherungswerte handelt.

\section{Erklärungshypothesen zur Motivation hagionymenbasierter HotelN-}

Wie lassen sich nun die eben dargestellten Befunde erklären? Wie Fahlbusch 2016: 26 ausführt, muss zwischen Namenbasis (hier: Hagionym) und Benennungsmotiv unterschieden werden. Die Motivation für einen einzelnen Hotelnamen bedarf natürlich eingehenderer Untersuchungen als die Feststellung der Namenbasis. Die Benennung eines Hotels nach einem Heiligen muss nicht unbedingt religiös motiviert sein. In vielen Fällen liegt dies lediglich in seiner geographischen Lage begründet und ist durch die Mikrotoponymie motiviert (cf. auch Herling 2012: 2017): eine nahe gelegene Kirche, ein hagionymenbasierter Straßenname oder ein Siedlungsnamen, der ein Hagionym enthält, kann den Ausschlag für die Benennung des Hotels gegeben haben, auch wenn heutzutage solche Benennungen weniger oft kreiert werden dürften.

Was z.B. Frankreich betrifft, so kann vor allem für Paris festgestellt werden, dass die Motivation für die Hotelbenennung im Straßennamen zu finden ist: Hôtel St. Honoré in der rue Saint-Honoré, Hôtel St. Michel auf dem boulevard Saint Michel; nach einem in der Nähe befindlichen Bauwerk oder auch nach Bauwerk und Straßennamen zusammen benannt ist beispielsweise das Hotel Saint-Charles Tour Eiffel (in der rue Saint-Charles, nahe des Eiffelturms). Ansonsten sind HotelN natürlich sehr oft auf Hagiotoponyme zurückzuführen, unabhängig davon, ob es sich um bekannte Orte (La Bastide de Saint-Tropez in Saint-Tropez; Mercure Mont Saint-Michel in der Nähe von Le Mont-Saint-Michel) oder kleine Dörfer handelt (Hotel Saint-Cyr in La Ferté-SaintCyr in der Region Val-de-Loire) oder um Heilige, die früher in der jeweiligen Region 
gewirkt haben (Saint Denis, der erste Bischof von Paris, nur in Île-de France vertreten). Diese Feststellungen dürften in ähnlicher Weise auch für die hagionymisch basierten Hotelnamen in Italien und Frankreich gelten.

Eine Hypothese, die sich bei genauerer Betrachtung jedoch als falsch erweist, bestünde darin, die im Land vorherrschende Konfession als Grund für die vergleichsweise hohe Frequenz der hier untersuchten HotelN zu sehen. Denn im Protestantismus spielen die Heiligen im Gegensatz zur katholischen Kirche keine Rolle. Italien, Frankreich und Spanien sind in religiöser Hinsicht vorwiegend katholisch geprägte Länder, und insbesondere hatten die Heiligen in Italien eine starke Stellung, die ihnen nördlich der Alpen nie zukam. Dennoch lässt sich mit dieser Annahme nicht erklären, warum in den überwiegend katholisch geprägten Gegenden von Deutschland und in Österreich ein erheblich geringerer Prozentsatz von HotelN auf ein Hagionym zurückgehen. Beispielsweise lässt sich in der Toponymie Deutschlands dieser konfessionelle und später systempolitische Gegensatz gut verfolgen: Nach Hengst (2016:138) sind im ostdeutschen Sprachraum aufgrund der früheren politischen Situation OrtsN mit Sankt auffällig selten, besonders im Vergleich zu Bayern und Baden-Württemberg. Die weitgehende Abwesenheit von HotelN in Rumänien, die auf Heiligennamen zurückgehen, ließe sich mit dieser Hypothese gleichfalls nicht erklären, denn in der orthodoxen Religion nehmen die Heiligen eine weitaus bedeutendere Stellung ein als im Protestantismus.

Möglicherweise ist das Vorhandensein bzw. die Abwesenheit von hagionymenbasierten Hotelnamen auf kulturspezifische Traditionen zurückzuführen. Wie Rieger (in diesem Band) ausführt, ist es in Italien durchaus üblich, eine bestimmte Kategorie von Lebensmittelprodukten nach Heiligen zu benennen, während das in Deutschland so gut wie nicht vorkommt. Apotheken (vgl. Fahlbusch 2016) werden dagegen in Deutschland sehr häufig nach Heiligen benannt.

\section{Conclusio und Desiderata}

Diese Überlegungen führen zum letzten Kapitel des Beitrags: Wie gezeigt wurde, lässt sich ein starker Gegensatz: katholische Romania (Frankreich, Italien, Spanien) vs. deutschsprachiger Raum und Rumänien im Hinblick auf hagionymenbasierte HotelN feststellen. Um festzustellen, ob die eben geäußerte Hypothese zutrifft, wäre zunächst zu überprüfen, ob HeiligeN als Basis von HotelN in Rumänien vor 1945 geläufiger waren und lediglich infolge des politisch bedingten Systemwechsels danach verschwanden. Interessanter dürfte es jedoch sein, in einer geographisch erweiterten Studie zu untersuchen, in welcher Frequenz gegenwärtig in welchen Ländern bzw. Regionen HeiligenN in HotelN vorkommen und dabei die sprachräumlichen Grenzen zu überschreiten. Ein gesamteuropäisches Panorama würde darüber Aufschluss geben, inwieweit kulturräumliche, sprachliche oder nationale Faktoren die Frequenz dieses Benennungstyps beeinflussen.

In diesem Zusammenhang könnte die Studie auch auf HotelN ausgeweitet werden, die nicht vom Vorhandensein der formalen Basis mit dem Adjektiv Sankt, Santo/ 
Santa, Sfânt/Sfânta ausgeht, sondern auch Hotelbenennungen mit semantisch religiösen Bezügen oder Konnotationen einbezieht (Regina Angelorum in Bozen).

Schlussendlich wäre natürlich nach der kommunikativen und pragmatischen Funktion von HotelN zu fragen. Es gibt -zumindest keine uns bekannte - Untersuchung zur Erwartungshaltung der Gäste gegenüber einem nach einem HeiligenN benannten Hotel oder allgemeiner gesprochen darüber, ob der Name eines Hotels Einfluss auf das Verhalten der Hotelgäste bei der Buchung des Hotels hat, oder ob er lediglich wie ein Produktname als Ausdruck der Wertvorstellungen einer Epoche (Ronneberger-Sibold 2012: „Brand names as offers of identity“) gewertet werden kann. Für Produktnamen ist die Situation grundverschieden, hier werden häufig vor der Lancierung eines neuen Produkts mit einem bestimmten Namen potenzielle KonsumentInnen zum neuen Namen befragt.

\section{Bibliographie}

Arx, W. von. 1970. Das Kosterrituale von Biburg. Freiburg: Universitätsverlag Freiburg Schweiz. Annuario Alberghi d'Italia.2005. Roma: Ente Nazionale Italiano per il Turismo.

Bugheşiu, A. 2013. Names of Transylvanian Hotels in the Context of Globalisation. In Name and Naming. Proceedings of the 3rd International Conference on Onomastics. Conventional/ Unconventional in Onomastics. O. Felecan (ed.), 81-90. Cluj-Napoca: Mega/Argonaut.

Debus, F. 2012. Namenkunde und Namengeschichte. Berlin: Erich Schmidt.

Fahlbusch, F. 2016. Maria, Heil der Kranken. Heilige in Apothekennamen. In Heiligenverehrung und Namengebung, K. Dräger, F. Fahlbusch und D. Nübling (eds.), 257-269. Berlin/Boston: De Gruyter.

Giese, W. 1970. Noms d'hôtels de tradition populaire en France. In Mélanges de linguistique et de philologie romanes, G. Matoré (ed.), 145-152. Paris: Klincksieck.

Greule, A. 2016. Heiligennamen in der volkssprachlichen Liturgie. In Heiligenverehrung und Namengebung, K. Dräger, F. Fahlbusch und D. Nübling (eds.), 293-301. Berlin/Boston: De Gruyter.

Hengst, K. 2016. Namen von Kirchenheiligen sowie Heiligen und Sankt in Siedlungsnamen im östlichen deutschen Sprachraum. In Heiligenverehrung und Namengebung, K. Dräger, F. Fahlbusch und D. Nübling (Eds.), 121-147. Berlin/Boston: De Gruyter.

Herling, S. 2012. Namen touristischer Unterkünfte auf Mallorca. In Onomastics goes Business: Role and Relevance of Brand, Company and Other Names in Economic Contexts, H. Wochele, J. Kuhn, und M. Stegu (eds.), 203-218. Berlin: Logos.

Hotel Guide Romania 2006. Bucharest: Ministry of Transports, Constructions and Tourism, National Authority of Tourism.

Koß, G. 2002. Namenforschung. Eine Einführung in die Onomastik. Tübingen: Niemeyer.

Krüger, S. 2015. Wer macht schon Urlaub im Hotel «Zum Fingernagel». Die Welt, 8.5.2015; www.welt.de/140656741 (konsultiert am 25.8.2017).

Marck, B. 2012. Antoine de Saint Exupéry. La soif d'exister (1900-1936). Paris: Larchipel.

Nübling, D., F. Fahlbusch und R. Heuser. ${ }^{2} 2015$. Namen. Eine Einführung in die Onomastik. Tübingen: Narr.

Platen, Ch. 1997. Ökonymie. Zur Produktnamen-Linguistik im Europäischen Binnenmarkt. Tübingen: Niemeyer. 
Ronneberger-Sibold, E. 2012. Brand names as offers of identity. An overview over the 20th century. In Onomastics Goes Business: Role and Relevance of Brand, Company and Other Names in Economic Contexts, H. Wochele, J. Kuhn und M. Stegu (eds.), 1-18. Berlin: Logos.

Serianni, L. 1978. Nomi d’alberghi. Lingua nostra 39: 56-62.

Wochele, H. 2007. Hotel Names in Italy and Romania - A Comparative Analysis. In Names in Commerce and Industry: Past and Present, L. Kremer und E. Ronneberger-Sibold (eds.), 317-329. Berlin: Logos.

Wochele, H. 2009. Regal, Royal, Republică: Synchronische und diachronische Aspekte der Benennung von Hotels am Beispiel von Italien und Rumänien. In People, Products and Professions. Choosing a Name, Choosing a Language. Fachleute, Firmennamen und Fremdsprachen, E. Lavric, F. Fischer, C. Konzett, J. Kuhn und H. Wochele (eds.), 309-322. Frankfurt/Main: Lang.

Wochele, H. 2017. Hotelnamen als Werbeträger: Gegenwärtige Tendenzen in der Romania und im deutschsprachigen Raum. In Werbesprache pluridisziplinär - Aktuelle Tendenzen in der romanistischen Werbesprachenforschung, A. Hennemann, A. Lobin, K. Plötner und C. Schlaak (eds.), 183-192, Berlin: Frank \& Timme.

\section{Für die Korpuserstellung verwendetete Internetadressen}

Booking.com: www.booking.com (letzter Zugriff 02.09.2017).

Holidaycheck: www.holidaycheck.de (letzter Zugriff 28.08.2017).

Pages jaunes: https://mesannuaires.pagesjaunes.fr/ (letzter Zugriff: 27.08.2017).

Páginas amarillas: https://www.paginasamarillas.es/search/alojamientos/all-ma/madrid/ all-is/madrid/all-ba/all-pu/all-nc/hoteles/all-ct/27?what=hoteles\&where=madrid (letzter Zugriff 28.08.2017).

Hotelier.de: www.hotelier.de (letzter Zugriff 18.08.2017).

Schnelle-Hotelsuche.at: http://www.schnelle-hotelsuche.at (letzter Zugriff 24.08.2017).

\section{Internetadressen}

https://de.wikipedia.org/wiki/St._Germain (letzter Zugriff 28.09.2017).

https://fr.wikipedia.org/wiki/Liste_des_h\%C3\%B4tels_class\%C3\%A9s_cinq_\%C3\%A9toiles_en_France (letzter Zugriff am 25.09.2017).

https://www.wko.at/site/hotelsterne/Kriterienkatalog-Hotelklassifizierung-2015-2020.pdf (letzter Zugriff 29.09.2017).

www.insee.fr/fr/statistiques/1283850\#documentation (letzter Zugriff am 23.08.2017).

Statistik Austria: https://de.statista.com/statistik/daten/studie/294862/umfrage/ anzahl-der-beherbergungsbetriebe-in-oesterreich/ (letzter Zugriff 18.08.2017). 